\title{
A Randomized Clinical Trial Comparing the Insertion Conditions and Pressor Responses of Two Airway Devices 'Combitube' and 'Laryngeal Mask Airway' During Elective Anaesthetic Procedures
}

\author{
Sumanth Kankanala ${ }^{1}$, Shanmugam Chockalingam ${ }^{2}$, Varaprasad Karnati ${ }^{3}$, Sridevi Sreerama ${ }^{4}$, Meghanath Reddy ${ }^{4}$ \\ ${ }^{1}$ Associate professor, Department of Anaesthesiology, Prathimalnstitue of Medical Sciences, Karimnagar, Telangana, ${ }^{2}$ Professor, Department of \\ Anaesthesiology, Sapthagiri Institute of Medical Sciences and Research Centre, Bengaluru, ${ }^{3}$ Registrar, Department of Anaesthesiology, ${ }^{4}$ Junior Consultant, \\ Continental Hospitals, Hyderabad
}

\section{Abstract}

Background: The major responsibility of an anaesthesiologist is management of airway so as to provide adequate ventilation to the patient by securing an unobstructed airway when general anaesthesia is administered. Advanced airway devices like Laryngeal Mask Airway (LMA) and Combitube are now considered as alternatives to endotracheal intubation for securing the airway and providing adequate ventilation even in difficult intubation and emergency situations. Objectives: To determine and compare the insertion conditions and pressor responses of two airway devices 'combitube and laryngeal mask airway' during elective anesthetic procedures. Subjects and Methods: 60 patients of age between 18-55 years, belonging to ASA grade I and II, scheduled for elective surgeries under general anaesthesia were included in the study.Patients with uncontrolled cardiovascular, respiratory, hepatic, renal diseases, morbid obesity, pharyngeal masses, history of chronic obstructive pulmonary diseases, oesophageal pathology were excluded in the study. All patients were allocated randomly by envelope method into two groups of 30 each, Group-L and Group- C.For patients in group L- airway was secured with laryngeal mask airway.Patients in group C- airway was secured with combitube. Results: Insertion conditions were better with LMA when compared to Combitube $(\mathrm{P}=0.00325)$. Mean heart rate of LMA group was less at 1,2,3,7 minutes compared to combitube group. Mean systolic blood pressure of LMA group is significantly less at 1,2,3,5,7,10, minutes than that of combitube group. Mean diastolic blood pressure of LMA group is significantly less at 1,2, 3 \& 5 minutes compared to combitube group. Conclusion: We concluded that the insertion conditions (ease of insertion) were better and the associated pressor responses were less with LMA than with combitube.Based on our conclusions, we can say that LMA is a better alternative device when compared to combitube in maintaining an intact airway. However, both can be safely used to conduct general anaesthesia for elective surgical procedures.

Keywords: Laryngeal mask airway, Combitube, Pressor response, Ease of insertion.

Corresponding Author: Dr. Shanmugam Chockalingam, Professor, Department of Anaesthesiology, Sapthagiri Institute of Medical Sciences and Research Centre, Bengaluru.

Received: April 2019

Accepted: May 2019

\section{Introduction}

The major responsibility of an anaesthesiologist is management of airway so as to provide adequate ventilation to the patient by securing an unobstructed airway when general anaesthesia is administered. As such, no anaesthesia is safe unless diligent efforts are devoted to maintain an intact functional airway.Endotracheal intubation is the overall accepted 'Gold standard of securing the airway and providing adequate ventilation. However, endotracheal intubation requires time, a skilled anaesthesiologist or appropriate instruments and adequate circumstances with respect to space and illumination. ${ }^{[1]}$

The pressor responses to laryngoscopy and endotracheal intubation are very well recognised since 1951. It is a sympathetic reflex provoked by stimulation of the airway leading to transitory, variable and unpredictable increases in blood pressureand heart rate. This may be hazardous as increased blood pressure in susceptible patients may lead to myocardial insufficiency or cerebrovascular accidents. ${ }^{[2]}$ Attenuation of pressor responses to manipulation of the airway has been practiced either by deepening the plane of anaesthesia, by the use of drugs known to obtund them or by using advanced airway devices.

Advanced airway devices like Laryngeal Mask Airway (LMA) and Combitube are now considered as alternatives to endotracheal intubation for securing the airway and providing adequate ventilation even in difficult intubation and emergency situations.

Laryngeal mask airway was designed by Dr.A.I.J.Brain in the year 1981. It was introduced into clinical practice in the year 1987, and it was found that the technique of insertion of LMA 5obviated the need for laryngoscopy, which was a major cause of the pressor responses. ${ }^{[3]}$

In the year 1987 Dr. MichealFrass invented another 
advanced airway device, the oesophageal tracheal combitube in an attempt to ease the method of securing an intact airway and obtund the use of laryngoscopy and associated complications. $^{[4]}$

The use of LMA and combitube have many advantages over endotracheal intubation like avoidance of laryngoscopy, ease of insertion, minimal pressor responses to insertion and removal, minimal rise in I.C.P, I.O.P.But, laryngeal mask airway has certain disadvantages like high chances of aspiration, regurgitation, vomiting, leak, and trauma.Combitube has also certain disadvantages like unsuccessful insertion, over inflation of cuff, available in only two custom made sizes, increased air way trauma. ${ }^{[5,6]}$

Many studies have been done to show the advantages and disadvantages of both these airway devices. But, a search through the literature does not reveal any study comparing both these airway devices either in the routine anaesthetic practice or emergency situations.

We have made an attempt to compare both these airway devices viz. combitube and laryngeal mask airway with respect to the insertion conditions and pressor responses in patients undergoing elective surgeries under general anaesthesia in our Institute.

\section{Objectives:}

To determine and compare the insertion conditions and pressor responses of two airway devices 'Combitube' and 'Laryngeal mask airway' during elective anaesthetic procedures.

\section{Subjects and Methods}

After obtaining Institutional Ethical Committee clearance and the patient consent the study was carried out on 60 patients posted for various types of elective surgical procedures at K. L. E's Hospital and MRC, District Hospital, Belgaum during the period from August 2003 to July 2004.

60 patients of age between 18-55 years, belonging to ASA grade I and II, scheduled for elective surgeries were included.

\section{Inclusion criteria:}

1. ASA group I and II

2. Age between 18 to 55 years of both sexes, scheduled for elective surgeries were included.

\section{Exclusion criteria:}

1. Uncontrolled cardiovascular, respiratory, hepatic (or) renal diseases.

2. Morbid obesity, pharyngeal masses.

3. History of chronic obstructive pulmonary diseases, oesophageal pathology.

A thorough preanesthetic evaluation was carried out in all the patients and procedure was explained in detail to all the patients (each group had 30 patients).Patients were allocated randomly by envelope method into 2 groups. Group L \& Group C.

Group L (LMA - 30 patients):Cuff inflated with 30ml air in males ( 4 no. LMA) and $20 \mathrm{ml}$ air in females ( 3 no. LMA) Group C (Combitube - 30 patients):Combitube 37FSA, oropharyngeal balloon inflated with $85 \mathrm{ml}$ of air and $10 \mathrm{ml}$ of air at distal oesophageal cuff.

All the patients were investigated preoperatively and the investigations Haemoglobin estimation, Urine examination: albumin, sugar and microscopic examination, Random blood Sugar, ECG, Chest x-ray, Blood urea were done.

All patients were premeditated 15 minutes prior to surgery with Inj. Glycopyrrolate $0.004 \mathrm{mg} / \mathrm{kg}-1$, Inj. Midazolam $0.005 \mathrm{mg} / \mathrm{kg}-1$, Inj. Fentanyl- lmcg.kg-1. Patients were then pre-oxygenated with $100 \% \mathrm{O} 2,3 \mathrm{~min}$. Patients were induced with Inj. Thiopentone sodium- 5mgkg-l and muscle relaxation was facilitated with inj.Suxamethonium 1.5 $\mathrm{mg} / \mathrm{kg}-\mathrm{l}$. After $1 \mathrm{~min}$ later, a 2\% lidocaine jelly was applied on the dorsal surface of LMA and then inserted and secured.If LMA insertion was unsuccessful after two attempts, the patients were withdrawn from the study.

In Group C,size 37FSA combitube was passed, if the ventilation is inadequate, unsuccessful after two attempts, the patients were withdrawn from the study.

Anaesthesia was maintained with $\mathrm{O} 2: \mathrm{N} 2 \mathrm{O}$ (50:50) and inj. Vecuronium bromide was used for muscle relaxation and volume control mode ventilation.At the end of procedure patients were adequately reversed with Inj. glycopyrrolate $0.008 \mathrm{mg} / \mathrm{kg}$ and inj. neostigmine $0.05 \mathrm{mg} / \mathrm{kg}$.LMA was removed after ascertaining that the patient was able to open his/her mouth on command. Cuff was not deflated for removal of LMA.Combitube was removed after deflating both the cuffs.

All data are reported as mean values \pm 2 SD. Statistical Analysis of the demographic data was done using chi-square test. Comparison between the groups was done using student ' $t$ ' test. A P value $<0.005$ was considered statistically significant.

\section{Results}

The present study was conducted to compare the insertion conditions and pressor responses of two airway devices 'combitube' and 'laryngeal mask airway' during elective anaesthetic procedures.60 patients between 18-55 years of both sexes belonging to ASA class I and II undergoing elective surgeries under general anaesthesia were included in this study.

[Table 1] Shows the demographic data of the patients and the airway device used to secure the airway in 2 groups. There was no significant difference in sex distribution age and body weight in the two groups.Table II: Shows the grades of insertion conditions of the LMA and combitube group.Insertion conditions are better with LMA when compared to combitube.

[Figure 1] Shows the mean heart rate just before intubation and at $1,2,3,5,7,10 \& 15$ minutes of LMA and combitube. The mean heart rate of LMA group is significantly less at 1,2,3,7 min compared to combitube group.Fig-2 Shows the systolic blood pressure of LMA and combitube group just before intubation and at 1, 2, 3, 5, 7, 10,15 minutes. Mean systolic blood pressure of LMA group is significantly less at $1,2,3,5,7,10$ mins than that of combitube group.Fig-3 Shows the diastolic blood pressure of combitube and LMA group, just before intubation and at 1,2, 3, 5, 7, $10 \& 15$ 
minutes. Mean diastolic B.P of LMA group is significantly less at 1, 23 and 5 min's compared to other group.

Table 1: Demographic data

Table 1: Demographic data
\begin{tabular}{|c|l|l|c|}
\hline Group & Group C & Group L & P-VALUE \\
\hline $\begin{array}{c}\text { Airway device } \\
\text { used }\end{array}$ & Combitube & LMA & \\
\hline Male/ female & $16 / 14$ & $20 / 10$ & 0.291 \\
\hline $\begin{array}{c}\text { Mean age (yrs) } \pm \\
\text { SD }\end{array}$ & $35.27 \pm 10.49$ & $40 \pm 9.59$ & 0.073 \\
\hline $\begin{array}{c}\text { Mean weight } \\
(\mathrm{kg}) \pm \text { SD }\end{array}$ & $56.93 \pm 10.05$ & $55.70 \pm 5.54$ & 0.55 \\
\hline
\end{tabular}

Table 2: Insertion conditions

\begin{tabular}{|c|c|c|c|c|}
\hline Grades & Combitube & $\%$ & LMA & $\%$ \\
\hline 1 & 6 & $20 \%$ & 18 & $60 \%$ \\
\hline 2 & 15 & $50 \%$ & 10 & $33.3 \%$ \\
\hline 3 & 9 & $30 \%$ & 2 & 6.7 \\
\hline \multicolumn{5}{|c|}{${ }^{*} \mathrm{P}=0.00325$} \\
\hline
\end{tabular}

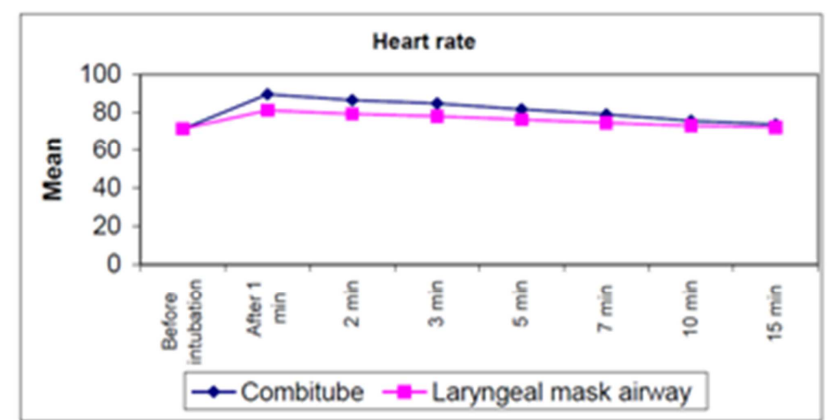

Figure 1: Association between HR and Airway device

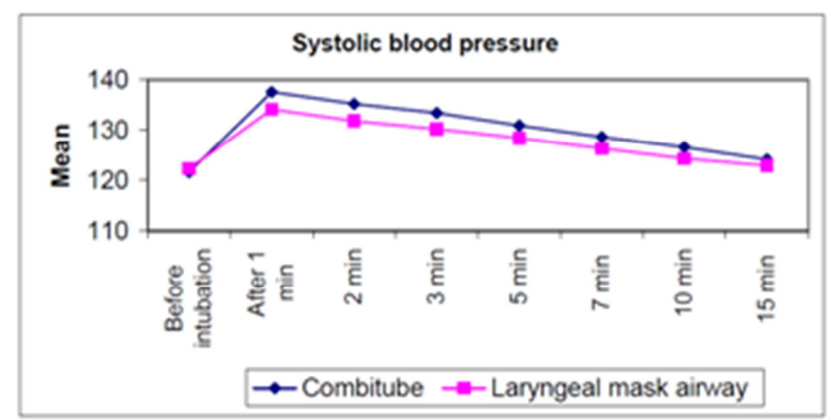

Figure 2: Association between SBP and Airway device

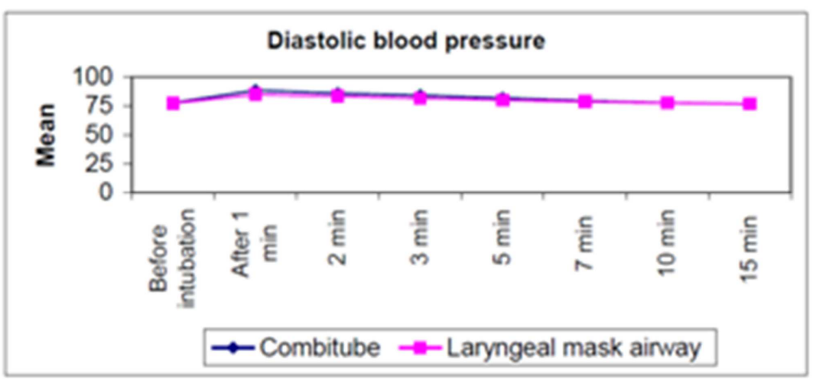

Figure 3: Association between SBP and Airway device

\section{Discussion}

Pressor responses to endotracheal intubation have been studied from the past and have shown that epipharyngeal and laryngeal stimulation caused by laryngoscopy have led to transient significant increase in heart rate, blood pressure and increase in plasma catecholamine levels. Hypertensive patients are prone to much greater pressor responses than normotensive patients and show higher increases in the level of plasma catecholamines.

Shribman et al concluded that the major cause of the sympathoadrenal response to tracheal intubation arises from stimulation of the supraglottic region by tissue irritation induced by direct laryngoscopy. Insertion of the tube through the vocal cords and inflation of the cuff in the infraglottic region should contribute very little additional stimulation. ${ }^{[8]}$

In an another study Hassan et al reported that, by activating proprioceptors, direct laryngoscopy induces arterial hypertension, tachycardia and increased catecholamine concentrations proportional to the intensity of the stimulus exerted against the base of the tongue. However, subsequent tracheal intubation should stimulate additional receptors in the larynx and the trachea, thus enhancing the pressor and epinephrine response. ${ }^{[9]}$

The use of laryngeal mask airway and combitube avoids the need for the laryngoscopy resulting in less painful stimulation of the airway, and hence lesserdegree of pressor response. ${ }^{[10]}$ Since there are very few studies comparing pressor responses to LMA insertion and combitube, the objective of this study was to compare pressor responses and insertion conditions of LMA and combitube in healthy adult patients.

In our study the demographic data of patients age, sex and body weight were similar in the two groups as shown in Table-I.It was observed that insertion conditions are better with LMA when compared to combitube as shown in table 2. $(p<0.00325)$. These results in our study were in correlation with the study done by Klein $\mathrm{H}$. et al.12 where he compared the ease of insertion and effectiveness of ventilation of LMA and combitube. He found that LMA was easier to insert. This they attributed to the wide spread use of LMA compared to combitube.In an another study, Parment J, et al. concluded that anaesthesia providers generally are more accustomed to the use of LMA than the ETC for difficult airway management and rescue ventilation because of the wide spread use of the LMA for general anaesthesia. ${ }^{[1]}$

In our study, the mean heart rates were compared just before intubation, 1, 2, 3, 5, 7, 10 and 15 minutes after intubation. The mean heart rate in group L is significantly less at 1, 2, 3, 5 and 7 minutes. The results obtained in our study were similar to those obtained by HolfgangOczenski et al,5 where in the increase in heart rate was attributed to the increase in plasma catecholamine concentrations following insertion of combitube.

The mean systolic blood pressure in group $\mathrm{L}$ was significantly less at 1, 2, 3, 5, 7 and $10 \mathrm{~min}$. The results in our study are similar with those observed by other studies. In the study conducted by HolfgangOczenski e tal,5 where in the pressor responses after insertion of combitube and LMA were compared in 75 patients were scheduled for routine urological and gynecological surgeries, and they found that the 2 devices could be easily and rapidly inserted and adequate ventilation and oxygenation provided. They concluded that insertion of combitube causes a pronounced 
stress response and precautions should be taken in patients at risk of hypertension, bleeding and in patients with cardiovascular disease. Further they remarked that Insertion of the combitube was associated with a significantly higher and longer lasting increase in SAP, DAP, MAP, HR and plasma catecholamine concentrating compared with insertion of the LMA.

The mean diastolic blood pressure in group (L) was significantly less at 1, 2, 3 and 5 mins. The results observed in our study were similar to those observed in other studies. Thus in our study we observed better insertion conditions and lesser pressor responses with use of LMA.5Thus, from our study we observed that the insertion conditions were better with LMA when compared to combitube and pressor responses were less with LMA when compared to combitube.

The advantages of using LMA over combitube i.e. easier and quicker placement, lesser pressor response to placement were confirmed in our study.The cardiovascular responses included by laryngoscopy and intubation were more than twice as high as those produced by the insertion of a LMA. ${ }^{[12]}$

Although, we have not compared heart rate changes in comparison with endotracheal intubation, literature reveals that there is upto $25 \%$ increase in mean heart rate after endotracheal intubation.5The combitube in our study is also associated with similar if not higher response.In our study placement of the combitube was always oesophageal.

Thus, we attribute our findings to a higher mechanical pressure on the tissues of the anterior pharyngeal region, possibly by the $85 \mathrm{ml}$ cuff of the combitube and the irritation of the oesophagus by the insertion of the device and subsequent inflation of the smaller blocking balloon. Similar observations were made, where they compared pressor and catecholamine stress responses to insertion of the combitube, laryngeal mask air way or tracheal intubation. ${ }^{[5]}$

It was also observed that insertion of the combitube was associated with a higher incidence of post-operative sore throat and dysphagia than tracheal intubation by direct laryngoscopy or insertion of the LMA. But, in emergency situations combitube was found to be better than LMA as the incidence of gastric aspiration is prevented by its distal balloon. Its use is well suited for emergency situations including unexpected difficult airway, failed tracheal intubation, when there is aspiration of gastric contents, upper air way bleeding or continued vomiting. ${ }^{[13]}$

\section{Conclusion}

We concluded that the insertion conditions (ease of insertion) were better and the associated pressor responses were less with LMA than with combitube.Based on our conclusions, we can say that LMA is a better alternative device when compared to combitube in maintaining an intact airway. However, both can be safely used to conduct general anaesthesia for elective surgical procedures.

\section{References}

1. Rushman GB, Davies NJH, Cashman JN. Intubation and ventilation. In: Rushman GB, Davies NJH, Cashman J N editors. Lee's synopsis of anaesthesia. Great Britain: MPG; 1999.246 - 253.

2. Derbyshire DR, Chimielewski A., Fell D, Vater M, Achola 1, Smith G. Plasma catecholamine responses to tracheal intubation. Br J. Anaesth, $1983 ; 55: 855-860$

3. KeerthiKumar.S, Lt. Cop Ramanaik. The history of evolution of laryngeal Mask airway. Indian J. Anaesth 1999;42:22-23.

4. Urtubia RM, Aguila CM, Cumsille M A. Combitube: A Study for proper use. AnesthAnalg 2000;90: 958-62.

5. HolfgangOczenski, MD, Herbert Krenn. Hemodynamic and Catecholamine stress responses to insertion of combitube, laryngeal mask airway or tracheal intubation. AnesthAnalg 1999; 88:1389-94.

6. Krafft P, Nikolic A, Frass M. Esophageal rupture associated with the use of the Combitube. AnesthAnalg 1998; 87:1457.

7. Tomori Z, Widdicombe JG. Muscular, Brochomotor and Cardiovascular Reflexes elicited by mechanical stimulation of the respiratory tract. J Physiol 1969;200:25-49.

8. Shribman AJ, Smith G, Achola J. Cardiovascular and catecholamine responses to laryngoscopy with and with out tracheal intubation. $\mathrm{Br} \mathrm{J}$ Anaesth 1987 ; 59: 295 -299.

9. Hassan H. G, EL Sharkawy TY, Renck H. Haemodynamic and catecholamine responses to laryngoscopy with and with out endotracheal Intubation. ActaAnaesthesiolScand 1991;35:442-447.

10. Yoshitaka Fujii, Hiroyoshi Tanaka, HidenoriToyooka. "Circulatory responses to laryngeal mask airway insertion or tracheal intubation in normotensive and hypertensive patients. Can J Anesth 1995;42:32-36.

11. Parmet J, Colonna- Ramano P, Horrow J, Miller F, Gonzales J, Rosen Berg H. The laryngeal mask airway reliably provides rescue ventilation in cases of unanticipated difficult tracheal intubation along with difficult mask ventilation. AnesthAnalg 1998;87:661-665.

12. Wilson IG, Fell D, Robinson SL and Smith G. Cardiovascular response to Insertion of laryngeal mask. Anesthesia 1992;47: 300-302.

13. Oczenski W, Krenn H,.Dahaba AA, Binder M, Schahawi-Kienzi EL. Kohout S. Complications following the use of the combitube, tracheal tube and laryngeal mask airway. Anaesthesia 1999;54:1161-1165.

Copyright: () the author(s), publisher. Academia Anesthesiologica Internationalis an Official Publication of "Society for Health Care \& Research Development". It is an open-access article distributed under the terms of the Creative Commons Attribution Non-Commercial License, which permits unrestricted non-commercial use, distribution, and reproduction in any medium, provided the original work is properly cited.

How to cite this article: Kankanala S, Chockalingam S, Karnati V, Sreerama S, Reddy M. A Randomized Clinical Trial Comparing the Insertion Conditions and Pressor Responses of Two Airway Devices 'Combitube' and 'Laryngeal Mask Airway' During Elective Anaesthetic Procedures. Acad. Anesthesiol. Int. 2019;4(1):100-103.

DOI: dx.doi.org/10.21276/aan.2019.4.1.23

Source of Support: Nil, Conflict of Interest: None declared. 